Trabajo seleccionado para su publicación por el jurado del Premio Estudios Financieros, formado por: don Alfonso Aguiló Pastrana, doña Milagros Antón López, don Fernando Checa García, don Ángel de Miguel Casas, doña Laura Rayón Rumayor y don Javier Manuel Valle López.

\title{
Un análisis
}

empírico de los

\section{determinantes de}

\section{la împlicación \\ académica del \\ estudiantado universitario}

\section{Extracto:}

La implicación del estudiantado se refiere a la atención, al interés y al esfuerzo que ponen los estudiantes durante el proceso de enseñanza-aprendizaje y que contribuye a su rendimiento académico. Un adecuado clima en el aula puede contribuir a mejorar esta implicación. Sin embargo, se sabe todavía relativamente poco respecto a los mecanismos específicos que explican la relación entre las actividades que lleva a cabo el profesorado en el aula y la implicación académica del estudiantado. El propósito de la presente investigación es analizar esta cuestión desde las premisas de la teoría de la autodeterminación. En concreto, a partir de datos obtenidos de 239 estudiantes universitarios españoles, demostramos que la satisfacción de las tres necesidades psicológicas básicas de competencia, autonomía y relaciones contribuye a la implicación del estudiantado y que su motivación intrínseca y su bienestar explican esta relación.

Palabras clave: implicación académica, teoría de la autodeterminación, necesidades psicológicas. 


\section{An empirical analysis of the determinants of the academic engagement of university students}

\begin{abstract}
:
Student engagement refers to students' attention, interest and effort during the learning process, and contributes to their academic performance and learning. An appropriate classroom climate may contribute to enhance student engagement. However, little is known about the specific mechanisms that explain the relationship between classroom climate and students' academic engagement. The main purpose of this study is to examine this issue from the premises of selfdetermination theory. Specifically, data from a sample of 171 spanish university students show that satisfying the three psychological needs for competence, autonomy and relatedness contributes to academic engagement, and that intrinsic motivation and well-being explain this relationship.
\end{abstract}

Keywords: academic engagement, self-determination theory, psychological needs.

\section{La implicación académica del} estudiantado es un objetivo de vital importancia para los educadores

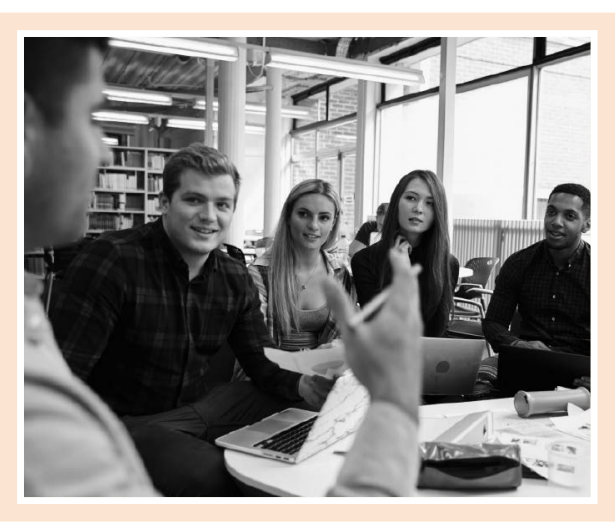

\section{INTRODUCCIÓN}

La implicación académica del estudiantado es un objetivo de vital importancia para los educadores, puesto que determina, en último término, el éxito académico de los alumnos y constituye un factor para medir el éxito de las intervenciones docentes en el aula (Elmore, 1990). Es por ello que su estudio ha centrado la atención de numerosos trabajos empíricos durante las últimas décadas.

Los investigadores han utilizado diferentes términos para hacer referencia a la implicación académica, tales como la «involucración», la «inversión psicológica», el «esfuerzo» o el «interés» del estudiantado. Todos estos vocablos hacen referencia, en mayor 0 menor medida, a la participación afectiva y comportamental del estudiantado y a su esfuerzo durante la experiencia de aprendizaje. Dada su contribución al desarrollo de los estudiantes y a sus logros académicos, no resulta sorprendente que los docentes e investigadores muestren un gran interés por conocer cómo fomentar dicha implicación durante las clases (Marks, 2000).

En este sentido, la implicación académica puede reforzarse a través de la instrucción académica y del modo en que el profesorado diseña las clases. Es, por tanto, una variable que se ve alterada por la forma en que el estudiantado responde a su entorno y, en particular, al clima existente en el aula (Csikszentmihalyi, 1990). Diversos autores afirman que, en la medida en que el profesorado sea capaz de proponer a sus alumnos tareas no rutinarias y suficientemente 
[...] la implicación académica puede reforzarse a través de la instrucción académica y del modo en que el profesorado diseña las clases

complejas y estimulantes que les permitan ejercer su autonomía en el aula y que creen un entorno de aprendizaje agradable, su implicación se verá mejorada (Csikszentmihalyi, 1990).

La teoría de la autodeterminación (Deci y Ryan, 1985) ofrece un marco teórico sólido y adecuado al estudio de los determinantes de la implicación académica, ya que analiza en qué medida los recursos internos del estudiantado interactúan con el clima en el aula para influir en la implicación académica de los alumnos. En concreto, en la presente investigación nos centramos en las premisas de la teoría de la satisfacción de las necesidades básicas para estudiar cómo influir en la implicación académica del estudiantado universitario.

Desde la teoría de las necesidades básicas existen tres necesidades inherentes a la vida humana, como son la necesidad de competencia, de autonomía y de relaciones. En la medida en que el estudiantado vea satisfechas estas tres necesidades durante el proceso de aprendizaje, su implicación académica se incrementará. Es decir, si el profesor es capaz de diseñar un proceso de enseñanza-aprendizaje que logre cubrir las necesidades de competencia, autonomía y relaciones de sus estudiantes, estos responderán con una mayor implicación académica. Sin embargo, los estudios existentes hasta la fecha no han logrado determinar con exactitud a través de qué variables se produce esta relación; es decir, existe una "caja negra» entre las dos variables, ya que investigaciones anteriores no han identificado cuáles son los mecanismos por los cuales la satisfacción de tales necesidades resulta en una mayor implicación académica, tal y como queda representado en la figura 1.

Nuestra investigación pretende contribuir, al menos en parte, a abrir esta "caja negra». En este sentido, la teoría de la autodeterminación propone que, en la medida en que el contexto de aprendizaje satisfaga las necesidades básicas del estudiantado, se contribuirá a un mayor crecimiento psicológico, a la internalización del aprendizaje y al bienestar de los estudiantes, que son las variables que determinan, en último término, la implicación académica (Van den Broeck, Ferris, Chang y Rosen, 2016). Siguiendo estas premisas, en la pre-

\section{[...] en la medida en que el contexto de aprendizaje satisfaga las necesidades básicas del estudiantado, se contribuirá a un mayor crecimiento psicológico, a la internalización del aprendizaje y al bienestar de los estudiantes}

Figura 1. Caja negra entre la satisfacción de las necesidades básicas y la implicación académica

Satisfacción de las necesidades básicas del estudiantado
Implicación académica 
sente investigación se analiza empíricamente en qué medida el crecimiento psicológico, la internalización del aprendizaje y el bienestar de los estudiantes son variables que intervienen (o median) en la citada relación entre la satisfacción de las necesidades básicas y la implicación académica. Así pues, nuestro propósito es indagar en el contenido de la citada caja negra para comprender mejor cómo se produce el incremento de la implicación académica a través de la acción del profesorado y del clima en el aula (véase figura 2).
Para contrastar nuestras hipótesis hemos llevado a cabo un estudio empírico en una muestra de estudiantes universitarios que cursan un grado en Administración de Empresas. Los resultados obtenidos indican que, en la medida en que en el aula se contribuya a satisfacer las necesidades básicas del estudiantado, este reportará una mayor implicación académica y que esta relación se puede explicar, al menos en parte, por el mayor crecimiento psicológico y el bienestar que experimentan los alumnos durante las clases.

\section{Figura 2. Abriendo la caja negra: variables intermedias entre la satisfacción de las necesidades básicas y la implicación académica}

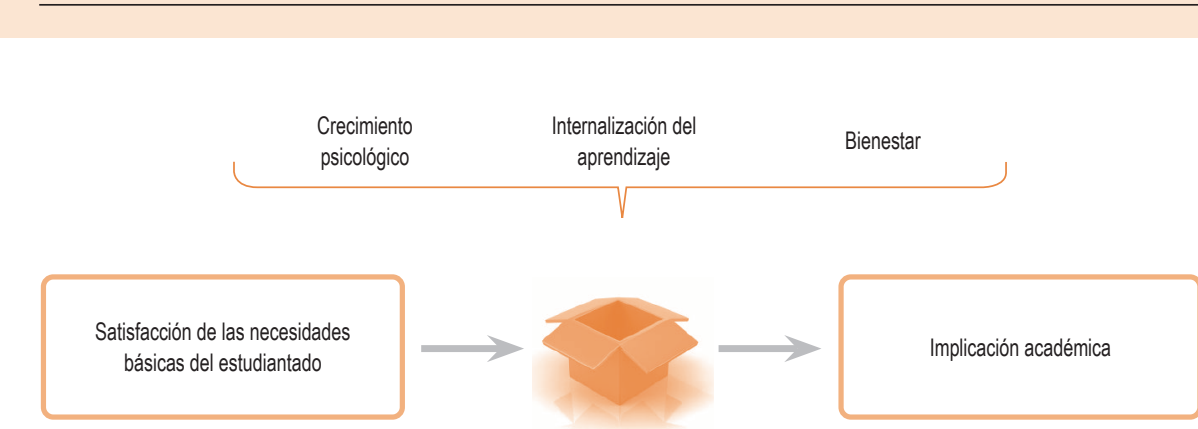

Fuente: elaboración propia

\section{SATISFACCIÓN DE LAS NECESIDADES PSICOLÓGICAS BÁSICAS E IMPLICACIÓN ACADÉMICA}

La implicación académica de los estudiantes durante las clases es uno de los factores que contribuyen en mayor medida al aprendizaje efectivo y a los buenos resultados académicos durante la época de la formación universitaria (Walker, Greene y Mansell, 2006). De hecho, autores tales como Trowler y Trowler (2010) llegan a afirmar que «el valor de la implicación académica es incuestionable» (p. 9).

La definición de «implicación académica» no resulta sencilla, ya que se trata de una especie de «metaconstructo» que aglutina ideas procedentes de diferentes líneas de investigación. Kahu (2013) diferencia entre tres enfoques para definir y entender la implicación académica:
- Enfoque conductual.

- Enfoque sociocultural.

- Enfoque psicológico.

Desde el «enfoque conductual», la implicación académica se define tanto en términos de las prácticas institucionales que se llevan a cabo en el centro educativo como en las respuestas del estudiantado en términos de satisfacción y logros. Algunos de los indicadores para medir la implicación académica desde este punto de vista son el tiempo dedicado por el estudiante a las tareas propuestas por el profesor, la integración social y académica, las prácticas docentes, los retos académicos, etc. 
El «enfoque sociocultural», por su parte, se centra en la influencia que tiene el contexto social del estudiantado en su experiencia de aprendizaje (por ejemplo, la disciplina existente en el centro educativo, la cultura académica, los valores de la sociedad, etc.). Sin embargo, uno de los problemas que presentan estas dos definiciones radica en la dificultad para discernir entre las prácticas llevadas a cabo de forma intencionada por el equipo educativo y las respuestas del estudiantado en términos de su implicación académica.

Finalmente, desde el «enfoque psicológico», el énfasis se pone en los procesos sociopsicológicos del estudiantado, que evolucionan a lo largo del tiempo y que varían en intensidad durante su etapa formativa. A diferencia del enfoque conductual y sociocultural, el enfoque psicológico permite diferenciar claramente entre la implicación académica y sus antecedentes, y ha sido la perspectiva más comúnmente adoptada en las investigaciones empíricas para definir este término. Siguiendo esta tendencia, en el presente trabajo se define la «implicación académica» como un proceso psicológico interno al estudiante que es maleable y que puede verse influido por el entorno.

La «implicación académica», desde el enfoque psicológico, es un concepto amplio que recoge tanto la dimensión comportamental como la dimensión cognitiva y la dimensión afectiva de la implicación académica del estudiantado. La dimensión comportamental incluye aspectos tales como conductas positivas durante el proceso de enseñanza-aprendizaje o la participación voluntaria en actividades extracurriculares, entre otros. La dimensión cognitiva se refiere a los esfuerzos y a la atención para lograr dominar los conocimientos o las habilidades propias de la disciplina. Finalmente, la dimensión afectiva hace referencia al vínculo emocional entre el estudiante y la experiencia de aprendizaje (por ejemplo, interés, disfrute, motivación, etc.).

Dada la relevancia de la implicación académica del estudiantado no es de extrañar que numerosas investigaciones se hayan preocupado por analizar cuáles son los medios que están a disposición del profesorado y que pueden utilizarse en el aula para favorecer dicha implicación. En este sentido, el estudio de los determinantes de la implicación académica se ha llevado a cabo desde diferentes marcos conceptuales, pero quizás la teoría de la autodeterminación (Deci y Ryan, 1985) sea el enfoque teórico que ha gozado de mayor popularidad entre los investigadores recientes del campo de la educación. La teoría de la autodeterminación parte de la idea defendida por las teorías contemporáneas de la motivación de que las personas inician y persisten en sus comportamientos en la medida en que consideran que dichos comportamientos contribuyen a alcanzar los objetivos deseables (por ejemplo, Vroom, 1964). Desde este marco teórico, para entender el modo en que se comportan las personas es necesario comprender cuáles son las necesidades que actúan como motivadoras del comportamiento. En otras palabras, si las personas muestran aquellos comportamientos que les permiten alcanzar los objetivos deseables en su vida es porque dichos objetivos contribuyen a satisfacer una serie de necesidades básicas que son inherentes a todo ser humano. Las tres necesidades psicológicas básicas según la teoría de la autodeterminación son la necesidad de competencia, de autonomía y de relaciones. Estas necesidades permiten mantener el crecimiento, la integridad y la salud de la persona.

La "competencia» hace referencia a la necesidad de sentirse eficaz en el logro de los propios objetivos y en las interacciones con el entorno; refleja el deseo del individuo de utilizar sus habilidades y de estar preparado para gestionar de un modo eficaz los retos externos que puedan presentársele. La «autonomía» se relaciona con la necesidad de iniciar y regular las propias acciones. Finalmente, las «relaciones» se vinculan a la necesidad de establecer lazos emocionales fuertes con los demás. Refleja el deseo de sentirse conectado emocionalmente y vinculado a unas relaciones sociales seguras y satisfactorias.

Diversos investigadores han aplicado estos mismos conceptos al ámbito de la educación, llegando a definir las «necesidades psicológicas del estudiantado» en los siguientes términos. En primer lugar, la «necesidad de competencia del estudiantado» se vincula al grado en que el estudiante percibe que las actividades de enseñanza-aprendizaje le plantean retos y contribuyen a desarrollar sus capacidades académicas, incrementando su sentimiento de autoeficacia. La «necesidad de autonomía del estudiantado» se satisface cuando las actividades propuestas en el aula por el profesorado ofrecen la posibilidad de percibir una sensación de libertad psicológica y de locus interno. La «necesidad de relaciones» se satisface cuando el estudiante se relaciona con otros estudiantes de una forma auténtica y basándose en el respeto y en el cuidado mutuo (Reeve, 2012). 


\section{Dentro de la teoría de la \\ autodeterminación, el enfoque de \\ las necesidades básicas explica \\ por qué determinados estudiantes \\ se implican en las actividades de \\ enseñanza-aprendizaje, mientras \\ que otros se muestran pasivos \\ durante las clases}

Dentro de la teoría de la autodeterminación, el enfoque de las necesidades básicas explica por qué determinados estudiantes se implican en las actividades de enseñanza-aprendizaje, mientras que otros se muestran pasivos durante las clases (Sheldon, Ryan y Reis, 1996). Este enfoque sugiere que, si las actividades planteadas por el profesorado satisfacen las tres necesidades psicológicas básicas de los estudiantes, estos mostrarán mayor energía, concentración y persistencia (es decir, mayor implicación académica). Esto quiere decir que los profesores que planteen tareas y actividades en el aula que permitan a los estudiantes sentirse más capaces, autónomos y seguros en las relaciones con sus compañeros lograrán incrementar la implicación académica de sus alumnos. Partiendo de estas premisas, formulamos nuestra primera hipótesis en los siguientes términos:

- Hipótesis 1. La satisfacción de las necesidades psicológicas se relaciona de forma positiva con la implicación académica del estudiantado.

\section{VARIABLES QUE EXPLICAN LA RELACIÓN ENTRE LA SATISFACCIÓN DE LAS NECESIDADES PSICOLÓGICAS BÁSICAS Y LA IMPLICACIÓN ACADÉMICA}

La teoría de la autodeterminación y el enfoque de las necesidades básicas proponen que el resultado de satisfacer las tres necesidades básicas del ser humano tiene como resultados inmediatos el crecimiento psicológico de la persona, una mayor internalización psicológica y un mayor bienestar (Deci y Ryan, 2000) (véase figura 3). El crecimiento psicológico supone que el individuo se implica en actividades que se consideren interesantes incluso en ausencia de estímulos o recompensas externas. En este sentido, el crecimiento psicológico se ha considerado como sinónimo de la motivación intrínseca (Van den Broeck et al., 2016). De forma similar, la internalización psicológica representa la tendencia del individuo a alejarse de formas de motivación extrínseca (es decir, manifestar un determinado comportamiento como consecuencia de la existencia de recompensas), mostrando formas de motivación que están más internalizadas e integradas con la propia persona. Por último, el bienestar se vincula al estado afectivo positivo de la persona, a su satisfacción con la vida o a su vitalidad, entre otros aspectos.
En cuanto a la primera de las relaciones propuestas en la figura 3, «la motivación intrínseca del individuo se verá favorecida por aquellas condiciones que conduzcan a la satisfacción de las necesidades psicológicas básicas» (Deci y Ryan, 2000, p. 233). En este sentido, diversas investigaciones han demostrado que la satisfacción de la necesidad de competencia resulta fundamental para promover la motivación intrínseca de las personas. En otras palabras, al proporcionar a un estudiante, por ejemplo, retroalimentación sobre sus logros académicos, el estudiante se sentirá más competente y, como resultado, estará más motivado con las tareas propuestas en el aula (Vallerand y Bissonnette, 1992). Además, autores tales como Deci y Ryan (2000) defienden la idea de que la autonomía en el desarrollo de las tareas académicas y las relaciones sociales son también factores críticos para lograr estudiantes con una mayor motivación intrínseca. Así pues, cabe esperar un efecto positivo entre la satisfacción de las tres necesidades psicológicas básicas y la motivación intrínseca. De forma similar, y según la idea de la internalización psicológica, cabe esperar que la relación 
sea negativa con la motivación extrínseca (segunda relación en la figura 3). En este sentido, Standage, Duda y Ntoumanis (2005) demostraron en una muestra de 950 estudiantes en institutos británicos que la satisfacción de las necesidades psicológicas básicas se relaciona de forma positiva con la motivación intrínseca (con un coeficiente de 0,94 ) y de forma negativa con la motivación extrínseca (coeficiente de - 0,50). Por último, por lo referente a la tercera de las relaciones que se muestran en la figura 3 , la teoría de la autodeterminación establece que "fluctuaciones en la satisfacción de las necesidades básicas predecirán fluctuaciones en el bienestar» (Deci y Ryan, 2000, p. 243). Por ejemplo, Reis et al. (2000) demostraron empíricamente que la autonomía y competencia percibida por los estudiantes están correlacionadas positivamente con su bienestar. En contextos más específicos, llardi, Leone, Kasser y Ryan (1993) demostraron que la satisfacción de las tres necesidades psicológicas básicas en los trabajadores de una muestra de empresas se relaciona de forma positiva con su autoestima y salud en general, mientras que Kasser y Ryan (1999) observaron que la satisfacción de estas necesidades contribuye al bienestar de una muestra de enfermeras. En línea con todo lo expuesto anteriormente, formulamos las hipótesis 2, 3 y 4 como sigue:
- Hipótesis 2. La satisfacción de las necesidades psicológicas se relaciona de forma positiva con la motivación intrínseca del estudiantado.

- Hipótesis 3. La satisfacción de las necesidades psicológicas se relaciona de forma negativa con la motivación extrínseca del estudiantado.

- Hipótesis 4. La satisfacción de las necesidades psicológicas se relaciona de forma positiva con el bienestar del estudiantado.

Además de las anteriores relaciones, varias investigaciones en el ámbito educativo han defendido la importancia que tienen la motivación intrínseca y extrínseca y el bienestar del estudiantado para explicar su implicación académica. En este sentido, trabajos tales como los de Boyd (2002) han demostrado que es más probable que estudiantes con una mayor motivación intrínseca persistan en sus esfuerzos durante el aprendizaje y al afrontar retos académicos. El concepto de «flow» es relevante para entender esta relación. Seligman y Csikszentmihalyhi (2000) definen flow como la «sensación holista que sienten las personas cuando actúan con una involucración total» (p. 36). Desde la perspectiva del flow una condición sine qua non para lograr una mayor implicación en una tarea determinada es la motivación intrínseca. El estado de flow es un estado agradable para el individuo, que se relaciona

Figura 3. Consecuencias inmediatas de la satisfacción de las necesidades psicológicas básicas

Satisfacción de las necesidades psicológicas básicas:

- Necesidad de competencia

- Necesidad de autonomía

- Necesidad de relaciones
Motivación intrínseca (crecimiento psicológico)

Motivación extrínseca (internalización psicológica)

Estados afectivos positivos (bienestar) 
directamente con la motivación intrínseca. Varias investigaciones empíricas han demostrado que individuos con mayores niveles de motivación intrínseca muestran también mayores niveles de implicación con las tareas que llevan a cabo, bien en el ámbito laboral o académico (por ejemplo, Kowal y Fortier, 1999). La motivación extrínseca es también una variable que contribuye a incrementar la implicación académica. En este sentido, autores tales como Steers, Mowday y Shapiro (2004) argumentan que las recompensas extrínsecas son en cierto modo necesarias para favorecer la persistencia y la implicación de los estudiantes en el proceso de aprendizaje-enseñanza. Por último, diversos trabajos han demostrado la influencia positiva que tiene el bienestar del estudiantado en su implicación académica (para una revisión sobre el tema, véase Bailey, Madden, Alfes y Fletcher, 2017). Por ejemplo, la teoría abierta de las emociones positivas (Fredrickson, 2001) afirma que las emociones positivas ayudan a ampliar el rango de pensamientos y acciones en la mente de los individuos, lo cual beneficia su implicación. Los trabajos de Salanova, Schaufeli, Xanthopoulou y Bakker (2010) sugieren que la experiencia de estados afectivos positivos incrementa la involucración en el trabajo. Por todo esto, planteamos las siguientes hipótesis en estos términos:

- Hipótesis 5. La satisfacción de las necesidades psicológicas se relaciona de forma positiva con la motivación intrínseca del estudiantado.
- Hipótesis 6. La satisfacción de las necesidades psicológicas se relaciona de forma negativa con la motivación extrínseca del estudiantado.

- Hipótesis 7. La satisfacción de las necesidades psicológicas se relaciona de forma positiva con el bienestar del estudiantado.

El razonamiento anterior sugiere que la satisfacción de las tres necesidades básicas del estudiantado contribuye a su implicación académica a través de tres variables mediadoras, como son la motivación intrínseca, la motivación extrínseca y el bienestar.

El siguiente y último bloque de hipótesis puede formularse como sigue:

- Hipótesis 8. La satisfacción de las necesidades psicológicas se relaciona de forma positiva con la motivación intrínseca del estudiantado.

- Hipótesis 9. La satisfacción de las necesidades psicológicas se relaciona de forma negativa con la motivación extrínseca del estudiantado.

- Hipótesis 10. La satisfacción de las necesidades psicológicas se relaciona de forma positiva con el bienestar del estudiantado.

La figura 4 resume de forma gráfica el modelo teórico que guía nuestra investigación, cuya metodología y resultados expondremos en los siguientes epígrafes. Como se observa en dicha figura, la satisfacción de las necesidades psicológicas básicas del estudiantado

Figura 4. Modelo teórico de la investigación

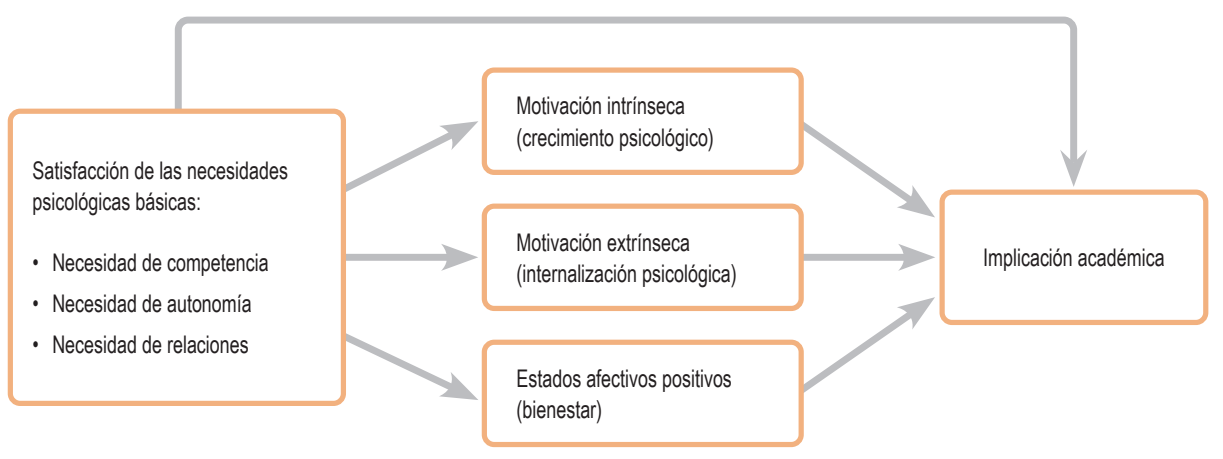

Fuente: elaboración propia. 
se relaciona de forma directa con su implicación académica (hipótesis 1). Además, incluimos tres variables que pueden explicar esta relación: motivación intrínseca, motivación extrínseca y bienestar. En este sentido, tras la revisión teórica realizada en este apartado, esperamos que la satisfacción de las necesidades psicológicas básicas influya de forma positiva sobre la motivación intrínseca y el bienestar del estudiantado y de forma negativa sobre su motivación extrínseca (hipótesis 2, 3 y 4). Estas tres variables, además, pueden contribuir a incrementar los niveles de implicación académica de los estudiantes (hipótesis 5, 6 y 7). De todo ello se desprende que la motivación intrínseca, la motivación extrínseca y el bienestar son variables intermedias en la relación entre la satisfacción de las necesidades psicológicas básicas y la implicación académica (hipótesis 8,9 y 10).

\section{METODOLOGÍA}

\subsection{Participantes}

La muestra para la presente investigación está compuesta de estudiantes del grado en Administración de Empresas de una universidad española matriculados en la asignatura Dirección de Empresas, materia obligatoria en el segundo curso del grado. Se invitó a los 285 alumnos matriculados en esta asignatura a participar en la investigación, completando un cuestionario distribuido por los autores del presente trabajo durante las horas de clase. Con el fin de favorecer la participación del estudiantado en la investigación, les aseguramos a todos los alumnos que sus respuestas iban a ser tratadas de forma confidencial y con fines exclusivamente académicos.

Un total de 239 estudiantes accedieron a participar, contestando a una batería de preguntas relativas a la satisfacción de sus necesidades psicológicas básicas, motivación intrínseca y extrínseca, bienestar e implicación académica, lo que supone una tasa de respuesta del $84 \%$. Se les pidió a los estudiantes participantes que indicaran su identificación académica en el cuestionario a fin de obtener una serie de datos académicos como variables de control, tal y como se detallará en el siguiente apartado.

\subsection{Medidas}

Las medidas de las variables utilizadas en la presente investigación se extrajeron de escalas publicadas con anterioridad. El apéndice incluye una descripción detallada de todas las medidas.

Para valorar el grado en que los estudiantes consideran que sus necesidades psicológicas están satisfechas, utilizamos la escala de Standage et al. (2005). Estos autores proponen tres subescalas correspondientes a la satisfacción de la necesidad de competencia, de autonomía y de relaciones, respectivamente, utilizando una escala Likert de 7 puntos (donde 1 significa totalmente en desacuerdo y 7 significa totalmente de acuerdo). Algunos ejemplos de esta escala son «Estoy muy formado en dirección de empresas», "Siento que tengo cierto grado de libertad de acción», «Me siento valorado por mis compañeros de la clase de Dirección de Empresas», etc. (véanse ítems 1 a 16 del apéndice).

La motivación intrínseca, extrínseca y el bienestar también se midieron utilizando las escalas de Standage et al. (2005) en una escala Likert de 7 puntos (véanse ítems 17 a 29 del apéndice).

Finalmente, para medir la implicación académica del estudiantado, utilizamos la escala de Marks (2000), compuesta de cuatro ítems. Estos ítems incluyen cuestiones relativas a la dimensión comportamental de la implicación académica (intensidad en el esfuerzo académico y realización de las tareas asignadas), a la dimensión cognitiva (grado en que el estudiante está atento) y a la dimensión afectiva (ausencia de aburrimiento durante las clases) (véanse ítems 30 a 33 del apéndice).

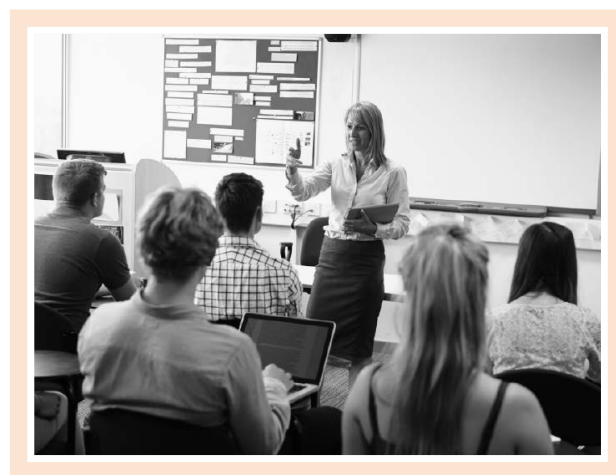


Por último, consideramos apropiado incluir una variable de control en nuestro modelo, como es la nota media del expediente académico, ya que diversos trabajos consideran que los logros académicos previos se relacionan de forma positiva con la implicación académica. Para ello, utilizamos la identificación académica proporcionada por los estudiantes participantes para extraer, a través de las bases de datos de la universidad, su nota media en el expediente académico hasta la fecha de realización de la presente investigación.

La tabla 1 muestra la matriz de covarianzas entre las variables incluidas en nuestra investigación.

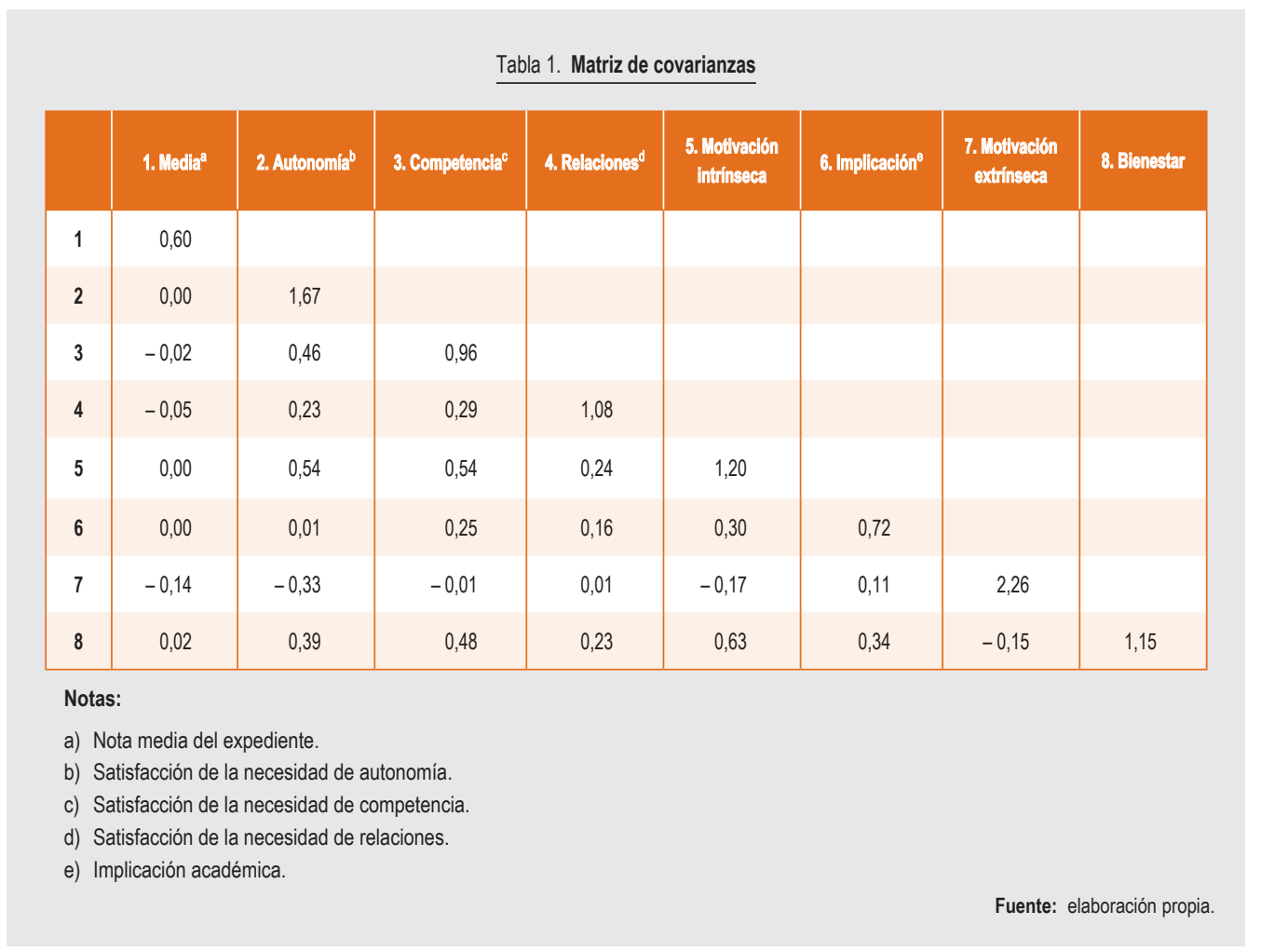

\subsection{Procedimiento estadístico}

Los análisis estadísticos se llevaron a cabo con los modelos de ecuaciones estructurales (SEM), utilizando para ello el programa EQS 6.3 para Windows, con el método de máxima verosimilitud. Antes de estimar los modelos estructurales necesarios para testar las hipótesis propuestas, llevamos a cabo unos análisis preliminares con la finalidad de valorar el riesgo del método común (Spector y Brannick, 2010).

A continuación, analizamos la dimensionalidad de los modelos de medida correspondientes a las escalas incluidas en la investigación (es decir, las relaciones entre las variables latentes y observables). Para ello, estimamos una serie de análisis factoriales confirmatorios (AFC) a fin de verificar la estructura propuesta de las escalas de medida.

La siguiente fase supone la estimación de los modelos estructurales que se corresponden con las hipótesis enunciadas en el apartado anterior, lo cual implica estimar modelos estructurales de mediación siguiendo el procedimiento de Baron y Kenny (1986). Este procedimiento implica estimar dos modelos estructurales. 


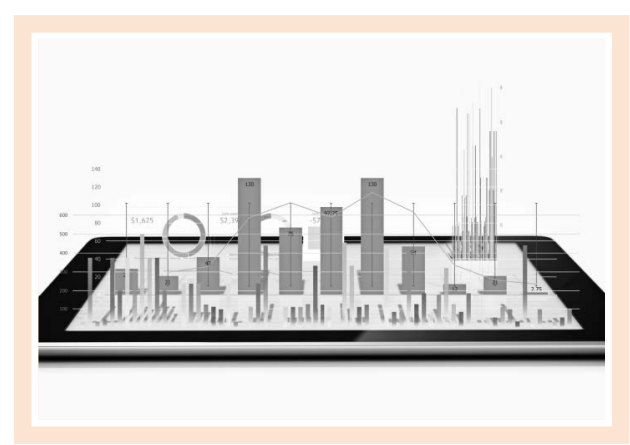

El primero de ellos se corresponde con un «modelo de efectos directos» que estima el efecto de las variables independientes sobre las dependientes. En el contexto de nuestra investigación, este modelo nos permitirá testar la hipótesis 1, ya que en él se estima el efecto directo de la satisfacción de las necesidades psicológicas básicas en la implicación académica. Para que exista mediación de otras variables en esta relación, los coeficientes estructurales del modelo de efectos directos tienen que ser estadísticamente significativos.

El segundo modelo estructural según el procedimiento de Baron y Kenny (1986) es el «modelo de mediación», que incluye también las variables intermedias (motivación intrínseca, motivación extrínseca y bienestar). La estimación de este modelo nos permitirá comprobar si se verifican las hipótesis 2 a 10. Asimismo, para verificar si el efecto mediador de estas tres variables intermedias en la relación principal (satisfacción de las necesidades psicológicas-implicación académica) es total, la relación «satisfacción de las necesidades psicológicas-implicación académica» del modelo de efectos directos deberá dejar de ser estadísticamente significativa en el modelo de mediación.

\section{RESULTADOS}

\subsection{Análisis preliminares}

Tal y como detallábamos en la sección anterior, antes de estimar los modelos estructurales valoramos el riesgo de sesgo del método común, ya que en nuestra investigación es un único informante (el estudiante) quien proporciona información acerca de todas las variables.
Este hecho puede sesgar los resultados, «inflando» las relaciones estimadas entre dos o más variables. Para valorar si existe este riesgo, llevamos a cabo el test del único factor de Harman (Podsakoff, MacKenzie, Lee y Podsakoff, 2003). Según este test, si existe riesgo de método común, emergería un único factor latente de todos los ítems incluidos en el cuestionario. Este test puede llevarse a cabo estimando un AFC que incluye todos los ítems de todas las variables consideradas en el estudio como indicadores de un factor latente que recoge la varianza común a todos estos ítems.

Los resultados de este AFC indican la ausencia de sesgo de método común, ya que los índices de ajuste están lejos de alcanzar los valores recomendados $\left(X^{2}{ }_{S B}=411,96 ; d . f .=104 ; p=0,00 ; B B N N F I=0,56\right.$; $\mathrm{CFI}=0,62 ; \mathrm{RMSEA}=0,13)^{2}$. Es decir, el hecho de que todas las variables se hayan valorado por la misma persona no debería afectar a los resultados obtenidos.

\subsection{Dimensionalidad de las escalas}

En cuanto a la escala de satisfacción de las necesidades psicológicas básicas, estimamos un AFC con las tres dimensiones de la escala (competencia, autonomía y relaciones) y sus indicadores (ítems 1 a 16). Este modelo incluye las tres dimensiones como factores latentes correlacionados. Atendiendo a las reducidas cargas factoriales de los ítems 3, 4 y 11 (inferiores a 0,4 ), decidimos eliminar estos ítems de los análisis posteriores. Los índices de ajuste del AFC modificado (tras eliminar estos tres ítems) muestran valores aceptables $\left(X_{S B}^{2}=73,68\right.$; d. f. $=59 ; p=0,09 ;$ BBNNFI = $=0,98 ; \mathrm{CFI}=0,99 ; \mathrm{RMSEA}=0,04)$, confirmando así la dimensionalidad propuesta de la escala.

Por lo relativo a las escalas de motivación intrínseca y extrínseca, cada una de ellas contiene tres ítems. La estimación de un AFC para cada una de ellas, por separado, nos lleva a un modelo saturado con cero grados de libertad, por lo que estimamos un AFC en el que la motivación intrínseca y extrínseca se consideran factores latentes correlacionados. Este modelo muestra un ajuste adecuado $\left(X_{S B}^{2}=8,76\right.$; d. f. = 8; $p=0,36 ;$ BBNNFI $=0,99 ; \mathrm{CFI}=0,99 ;$ RMSEA $=0,02$ )

\footnotetext{
${ }^{2} \mathrm{X}_{\mathrm{SB}}^{2}$ (chi cuadrado Satorra-Bentler); BBNNFI (Bentler-Bonett non-normed fit index); CFI (comparative fix index) y RMSEA (room mean square error of aproximation).
} 
e indica que los tres ítems de cada escala tienen cargas factoriales estadísticamente significativas en sus escalas correspondientes, con valores en todos los casos superiores a 0,6 .

El AFC para la escala de bienestar también muestra un buen ajuste $\left(X_{S B}^{2}=2,75 ;\right.$ d. f. $=2 ; p=0,25 ; B B N N F I=$ $=0,99 ; \mathrm{CFI}=0,99 ; \mathrm{RMSEA}=0,05$ ), lo cual confirma que todos estos ítems pertenecen a un único factor, con una alta fiabilidad, ya que las cargas factoriales son superiores a 0,6 en todos los casos.

Finalmente, nuestros análisis no permiten verificar la unidimensionalidad de la escala de la implicación académica, ya que el AFC muestra unos índices de ajuste lejos de los valores recomendados $\left(X_{S B}^{2}=8,66\right.$; d. $\mathrm{f}=2 ; \mathrm{p}=0,01 ; \mathrm{BBNNFI}=0,71 ; \mathrm{CFI}=0,89 ; \mathrm{RMSEA}=$ $=0,14)$ y unas cargas factoriales reducidas para los ítems $30(0,46), 32(0,29)$ y $33(0,42)$. Por esta razón, consideramos apropiado analizar por separado los cuatro ítems de la escala de la implicación académica (ítems 30 a 33) en los análisis posteriores. Por ello, diferenciaremos las relaciones propuestas en las hipótesis para cuatro variables correspondientes a la intensidad de los esfuerzos de los estudiantes (ítem 30, «intensidad»), al grado en que el estudiante realiza las tareas que le son asignadas (item 31, «tareas»), al grado en que el estudiante está atento en clase (ítem 32, «atención») y a la ausencia de aburrimiento durante las clases (ítem 33, «no aburrimiento»). Así pues, las hipótesis correspondientes al modelo representado en la figura 4 se estimarán considerando como variables dependientes estos cuatro conceptos de la implicación académica en lugar de una única variable.

\subsection{Modelos estructurales}

En cuanto a la estimación de los modelos estructurales correspondientes a nuestras hipótesis, la $\mathrm{X}^{2}$ para el «modelo de efectos directos» (véase modelo 1 en la tabla 2) no es estadísticamente significativo y los índices de ajuste están dentro de los valores aceptables $\left(X_{S B}^{2}=1,72 ; d\right.$. f. $=1 ; p=0,19 ;$ BBNNFI = 0,88; $\mathrm{CFI}=0,99 ; \mathrm{RMSEA}=0,06)$. Atendiendo a estos valores, nuestros resultados confirman que, una vez controlado el efecto de la nota media del expediente sobre la implicación académica, el valor de los coeficientes estructurales correspondiente a la influencia de la satisfacción de las necesidades psicológicas básicas sobre las cuatro variables de la implicación académica es estadísticamente significativo, con un valor de 0,17 para «intensidad», de 0,14 para «tareas» (al nivel del 10\%), de 0,24 para «atención» y de 0,20 para «no aburrimiento», tal y como muestra la solución estandarizada del modelo (véanse tabla 2 y figura 5). Así pues, se da la primera condición del procedimiento de Baron y Kenny (1986) para continuar analizando los efectos intermedios en esta relación, ya que los coeficientes en el modelo de efectos directos son estadísticamente significativos.

La estimación del «modelo de mediación» (véase modelo 2 en la tabla 2) también muestra un buen ajuste, atendiendo al valor de la $X^{2} y$ de los índices de ajuste $\left(X_{S B}^{2}=2,35 ;\right.$ d. f. $=4 ; p=0,67 ; B B N N F I=1 ; C F I=1$; RMSEA $=0,00$ ). Si nos fijamos en los valores de los coeficientes estructurales de este modelo, observamos que la satisfacción de las necesidades psicológicas básicas tiene una influencia positiva y significativa en la motivación intrínseca del estudiantado $(0,53)$, confirmando así la hipótesis 2, y sobre su bienestar $(0,44)$, lo cual confirma también la hipótesis 4. Sin embargo, no observamos que la relación con la motivación extrínseca sea estadísticamente significativa, por lo que nuestros datos no permiten corroborar la hipótesis 3 .

Por lo relativo a la influencia de las variables mediadoras en la implicación académica, la tabla 2 y la figura 5 muestran que la motivación intrínseca ejerce una influencia positiva y significativa sobre la «atención» $(0,25)$, lo cual permite corroborar la hipótesis 5 solo parcialmente, ya que su relación con las otras tres variables de la implicación académica no es estadísticamente significativa. La motivación extrínseca influye significativamente sobre las variables «tareas» $(0,17)$, aunque, de forma contraria a lo esperado, esta variable ejerce una influencia negativa con la variable «no aburrimiento» $(-0,19)$. En este caso, también, la hipótesis 6 se ve solo confirmada parcialmente por nuestros datos. Finalmente, en cuanto al efecto que tiene el bienestar del estudiantado sobre su implicación académica, observamos que esta variable ejerce un efecto positivo y significativo sobre las variables «intensidad» $(0,27)$, «atención» $(0,25)$ y «no aburrimiento» $(0,19)$, confirmando parcialmente la hipótesis 7 .

Atendiendo a estos resultados y a los valores de los efectos indirectos proporcionados por el programa EQS 6.3 podemos concluir que la motivación intrínseca del estudiantado y su bienestar son variables que ex- 
plican la relación entre la satisfacción de las necesidades psicológicas básicas y la implicación académica, aunque cabe diferenciar entre los distintos conceptos que recoge esta variable. En particular, la motivación intrínseca media la relación entre la satisfacción de las necesidades psicológicas básicas y las «tareas», mientras que el bienestar es una variable mediadora entre la satisfacción de las necesidades psicológicas básicas y la «intensidad», la «atención» y el «no abu- rrimiento» de los estudiantes. Por esta razón, las hipótesis 8 y 10 pueden confirmarse solo parcialmente.

Además, observamos que cuando las tres variables intermedias se incluyen en la ecuación (en el modelo de mediación), el efecto directo de la satisfacción de las necesidades psicológicas básicas sobre la implicación académica deja de ser significativo, lo cual evidencia la mediación total de la motivación intrínseca y del bienestar del estudiantado en la relación principal.

\section{Tabla 2. Parámetros estimados y errores estándar (entre paréntesis) de los modelos}

\begin{tabular}{|c|c|c|}
\hline & $\begin{array}{c}\text { Modelo } 1 \\
\text { Parámetros }\end{array}$ & $\begin{array}{c}\text { Modelo } 2 \\
\text { Parámetros }\end{array}$ \\
\hline 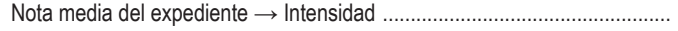 & $0,03(0,07)$ & $0,05(0,07)$ \\
\hline Nota media del expediente $\rightarrow$ Tareas & $0,01(0,06)$ & $0,12(0,06)$ \\
\hline Nota media del expediente $\rightarrow$ Atención & $0,02(0,07)$ & $0,03(0,06)$ \\
\hline 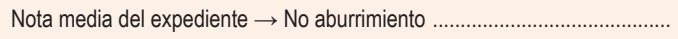 & $-0,02(0,09)$ & $-0,01(0,09)$ \\
\hline Satisfacción de las necesidades básicas $\rightarrow$ Intensidad .............................. & $0,17(0,11)^{\star *}$ & $0,04(0,13)$ \\
\hline 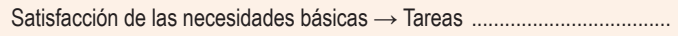 & $0,14(0,09)^{*}$ & $0,03(0,11)$ \\
\hline 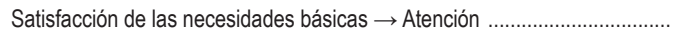 & $0,24(0,11)^{\star * *}$ & $0,00(0,12)$ \\
\hline 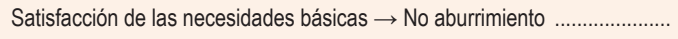 & $0,20(0,15)^{* *}$ & $0,05(0,17)$ \\
\hline Satisfacción de las necesidades básicas $\rightarrow$ Motivación intrínseca .............. & & $0,53(0,09)^{* * *}$ \\
\hline Satisfacción de las necesidades básicas $\rightarrow$ Motivación extrínseca ............. & & $-0,08(0,14)$ \\
\hline 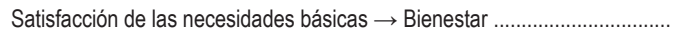 & & $0,44(0,14)^{* * *}$ \\
\hline 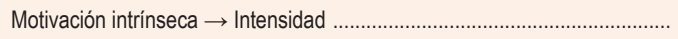 & & $0,04(0,10)$ \\
\hline 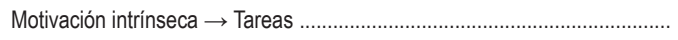 & & $0,12(0,09)$ \\
\hline 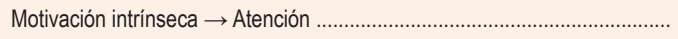 & & $0,25(0,09)^{* * *}$ \\
\hline 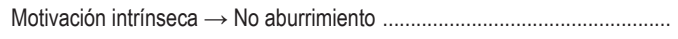 & & $0,10(0,13)$ \\
\hline 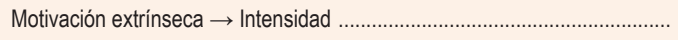 & & $0,09(0,06)$ \\
\hline 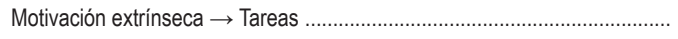 & & $0,17(0,05)^{* *}$ \\
\hline Motivación extrínseca $\rightarrow$ Atención & & $0,06(0,05)$ \\
\hline 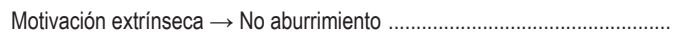 & & $-0,19(0,08)^{* * *}$ \\
\hline
\end{tabular}




\section{Tabla 2. Parámetros estimados y errores estándar (entre paréntesis) de los modelos (cont.)}

\begin{tabular}{|c|c|c|}
\hline & $\begin{array}{c}\text { Modelo } 1 \\
- \\
\text { Parámetros }\end{array}$ & $\begin{array}{c}\text { Modelo } 2 \\
- \\
\text { Parámetros }\end{array}$ \\
\hline \multicolumn{3}{|l|}{$\ldots / \ldots$} \\
\hline Bienestar $\rightarrow$ Intensidad & & $0,27(0,10)^{* * *}$ \\
\hline Bienestar $\rightarrow$ Tareas & & $0,14(0,08)$ \\
\hline Bienestar $\rightarrow$ Atención & & $0,25(0,09)^{\star * *}$ \\
\hline Bienestar $\rightarrow$ No aburrimiento & & $0,19(0,13)^{\star *}$ \\
\hline
\end{tabular}

Nota. Parámetros estandarizados; errores estándar entre paréntesis; ${ }^{*} p<0,10 ;{ }^{* *} p<0,05 ;{ }^{* * *} p<0,01$.

Fuente: elaboración propia

\section{Figura 5. Resultados}

\section{Modelo de efectos directos}

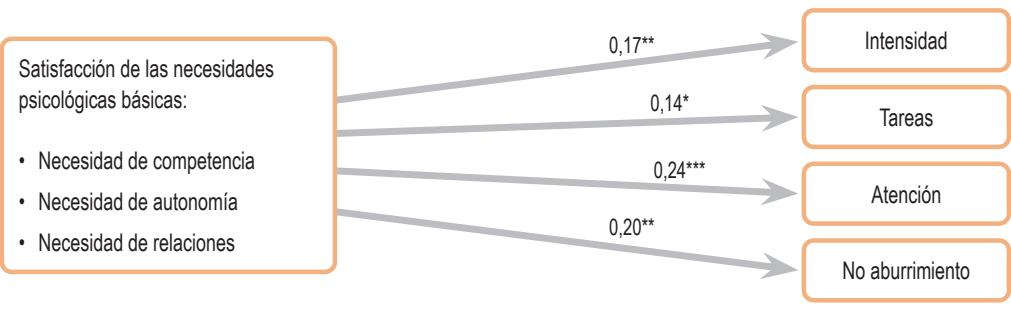

Modelo de mediación

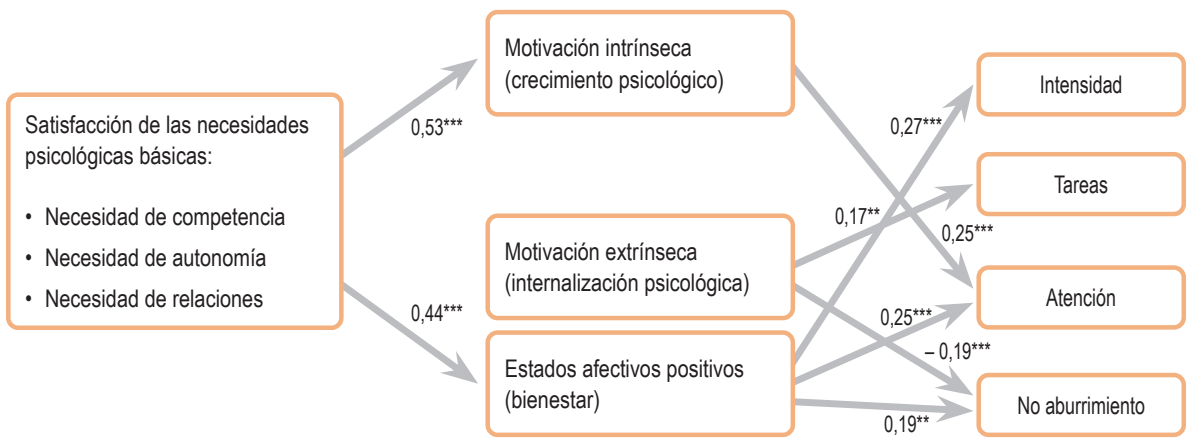

Fuente: elaboración propia 


\section{CONCLUSIONES}

Muchos docentes del ámbito universitario se plantean en la actualidad cómo favorecer la implicación académica de sus estudiantes, atendiendo al creciente número de distractores que pueden interferir en el proceso de enseñanza-aprendizaje (por ejemplo, falta de interés en la materia, uso de móviles en el aula, fatiga, amplia información sobre la materia en internet, etc.). La implicación académica es una variable de vital importancia en el proceso de aprendizaje del estudiantado, ya que se ha demostrado que contribuye ampliamente a su éxito académico.

Así pues, el propósito de esta investigación era analizar cómo favorecer la implicación académica de los estudiantes universitarios. En particular, nos planteábamos examinar la influencia que tiene la satisfacción de las necesidades psicológicas básicas del estudiantado propuestas por la teoría de la autodeterminación sobre su implicación académica. Tras la estimación de una serie de modelos de ecuaciones estructurales sobre datos obtenidos a partir de una muestra de estudiantes de una universidad española, podemos concluir que, en la medida en que se satisfagan las necesidades de competencia, autonomía y relaciones de los estudiantes en el aula, estos mostrarán una mayor implicación académica. Además, observamos que esta relación puede explicarse por diferentes estados afectivos y motivacionales, dependiendo de la variable de implicación que se tome en consideración.

\subsection{Implicaciones académicas}

Más concretamente, podemos extraer una serie de conclusiones valiosas acerca de la influencia que tiene el contexto de aprendizaje sobre algunos de los componentes de la implicación académica. Retomando la idea de que la implicación académica tiene un componente comportamental, un componente cognitivo y un componente afectivo, estructuraremos las conclusiones de nuestro trabajo atendiendo a estos tres componentes.

En cuanto al «componente comportamental» de la implicación académica (medido a través de la intensidad de sus esfuerzos y de la realización de las tareas), nuestros análisis demuestran que, en la medida en que el estudiante vea satisfechas sus necesidades de competencia, autonomía y relaciones en el aula, se producirá un incremento de su esfuerzo durante las clases y de su implicación con la realización de las tareas propuestas por el profesorado. No obstante, observamos que los procesos a través de los cuales se produce esta relación varían en función del indicador de implicación académica que se tome en consideración. En relación con la intensidad de los esfuerzos del estudiantado, la influencia de la satisfacción de las necesidades psicológicas básicas se ejerce a través del bienestar del estudiantado. Es decir, si la satisfacción de las necesidades psicológicas básicas repercute en estudiantes que se esfuerzan y ponen todo de su parte en el proceso de aprendizaje de una materia es porque estos estudiantes experimentan unos procesos afectivos positivos durante las clases (es decir, están satisfechos, contentos, relajados, etc.). En general, hay pocos trabajos que relacionen los procesos afectivos con los resultados académicos de los estudiantes. Nuestros resultados suponen un primer paso en esta dirección al demostrar que estudiantes con mayor bienestar se esforzarán más durante el proceso de enseñanzaaprendizaje. Esta conclusión está en la línea de las propuestas de las teorías de la atribución, las cuales predicen que las emociones de los estudiantes influyen en su persistencia en comportamientos relevantes para el aprendizaje.

No observamos, sin embargo, que la motivación extrínseca explique la relación entre la satisfacción de las necesidades psicológicas básicas y el esfuerzo, confirmando así las propuestas de diversos autores (por ejemplo, Angrist y Lavy, 2009) de que las variables motivadoras externas no son relevantes para incrementar la intensidad de los esfuerzos de los estudiantes. No es necesario proporcionar a los estudiantes recompensas externas (por ejemplo, ofrecer puntos adicionales en la nota final, reconocimiento público por parte del profesor, premios, etc.) para lograr que estos se esfuercen más. Sin embargo, estas

\section{[...] la implicación académica tiene un componente comportamental, un componente cognitivo y un componente afectivo}


recompensas sí que son importantes para lograr que los estudiantes realicen las tareas propuestas por el profesorado, ya que nuestros análisis demuestran que la motivación extrínseca media la relación entre la satisfacción de las necesidades psicológicas básicas y la realización de las tareas.

En cuanto al «componente cognitivo» de la implicación académica (medido en nuestra investigación a través del grado de atención que prestan los estudiantes durante las clases), observamos que la satisfacción de las necesidades psicológicas básicas determina la atención del estudiantado universitario. Una preocupación común entre los profesores universitarios en la actualidad es que sus estudiantes se distraen con mucha facilidad durante las clases, realizando todo tipo de actividades que poco tienen que ver con la materia que se está impartiendo (por ejemplo, preparando trabajos para otras asignaturas, consultando el ordenador 0 el móvil, etc.). Acaparar la atención del estudiantado es un objetivo para cualquier profesor, ya que garantiza el buen desarrollo de las clases y, en último término, juega un papel fundamental en el aprendizaje de los alumnos. Atendiendo a los resultados de nuestra investigación, diseñando actividades de enseñanzaaprendizaje y generando un clima en el aula que haga que los estudiantes se sientan competentes, autónomos y seguros en sus relaciones con los demás estudiantes será posible incrementar la atención que presten estos estudiantes durante las clases. Además, atendiendo a nuestros resultados, esta relación se explica por la motivación intrínseca y el bienestar de los estudiantes.

Por último, y en relación con el «componente afectivo» de la implicación académica (medido como la ausencia de aburrimiento), observamos que la satisfacción de las necesidades psicológicas básicas se relaciona de forma positiva con esta variable. Es decir, estudiantes que vean satisfechas estas tres necesidades básicas se aburrirán menos que estudiantes que no lo hagan. El aburrimiento del estudiantado es un tema relevante en el ámbito de la educación, ya que reduce los logros académicos y la satisfacción con la escuela o la universidad, derivando en altas tasas de abandono escolar. Así, nuestros resultados confirman las sugerencias proporcionadas por diversos autores acerca de la importancia de llevar a cabo actividades durante las clases que reduzcan el aburrimiento de los alumnos, que se basen en el diseño de estrategias de aprendizaje estimulantes y que favorezcan un clima de colaboración entre los estudiantes. Esta relación se produce, en nuestra muestra de estudiantes, a través del bienestar de los mismos.

Otro resultado interesante hace referencia a la relación negativa entre la motivación extrínseca y la ausencia de aburrimiento. En otras palabras, aquellos estudiantes que estén más motivados por variables externas tenderán a aburrirse más durante las clases. Este resultado es consistente con la teoría de la consecución de objetivos (Seifert, 2004) y corrobora resultados obtenidos en investigaciones previas (por ejemplo, Ntoumanis, 2001).

\subsection{Limitaciones y líneas futuras de inves- tigación}

Una de las limitaciones de nuestro trabajo se refiere a la obtención de datos, que en nuestro caso hemos obtenido a través de un único informante (el estudiante). Sería conveniente complementar esta información con la opinión que tiene el profesorado acerca de la implicación académica de sus alumnos como una forma de mejorar la fiabilidad de las medidas utilizadas en esta investigación.

Asimismo, nuestra investigación es de corte transversal y creemos que es interesante la realización de estudios longitudinales en los que la información sobre las variables se obtenga en diferentes momentos del tiempo, ya que es posible, por ejemplo, que la influencia de la satisfacción de las necesidades psicológicas básicas sobre la implicación académica del estudiantado no sea inmediata.

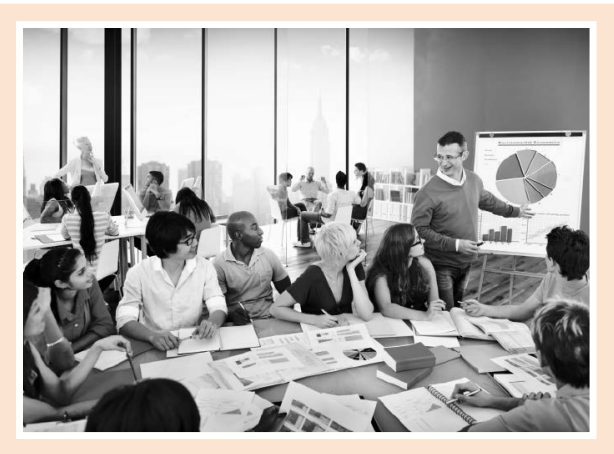


De forma contraria a lo observado por trabajos anteriores (por ejemplo, Marks, 2000), no observamos que los logros académicos previos del estudiantado (medido como la nota media del expediente) ejerzan ninguna influencia sobre la implicación académica. El background personal como predictor de la implicación académica es una cuestión que merece una mayor atención en trabajos futuros. Por ejemplo, sería interesante analizar la influencia del género o del apoyo social en la implicación académica.

\section{BIBLIOGRAFÍA}

Angrist, J. D. y Lavy, V. (2009). The effects of high stakes high school achievement awards: evidence from a randomized trial. American Economic Review, 99, 1.384-1.414.

Bailey, C., Madden, A., Alfes, K. y Fletcher, L. (2017). The meaning, antecedents and outcomes of employee engagement: a narrative synthesis. International Journal of Management Reviews, 19, 31-53.

Baron, R. y Kenny, D. (1986). The moderator-mediating variable distinction in social-psychological research. Journal of Personality and Social Psychology, 51, 1.173-1.182.

Boyd, F. B. (2002). Motivation to continue: enhancing literacy learning for struggling readers and writers. Reading and Writing Quarterly: Overcoming Learning Difficulties, 18, 257-277.

Broeck, A. van den, Ferris, D. L., Chang, C. H. y Rosen, C. C. (2016). A review of self-determination theory's basic psychological needs at work. Journal of Management, 42, 1.195-1.229.

Csikszentmihalyi, M. (1990). Flow: The Psychology of Optimal Experience. New York: Harper and Row.

Deci, E. L. y Ryan, R. M (1985). Intrinsic Motivation and Self-Determination in Human Behavior. New York: Plenum.

Deci, E. L. y Ryan, R. M. (2000). The «what» and "why» of goal pursuits: human needs and the self-determination of behavior. Psychological Inquiry, 11, 227-268.

Elmore, R. F. (1990). Restructuring Schools: The Next Generation of Educational Reform. San Francisco: Jossey-Bass.
Por último, consideramos necesario completar esta investigación incluyendo información sobre el rendimiento académico del estudiantado (por ejemplo, nota final obtenida en la asignatura) a fin de observar si el efecto de la implicación académica sobre el rendimiento es estadísticamente significativo. No obstante, esta información no ha sido posible incluirla en la presente investigación, ya que, a la fecha de finalización de la misma la nota final de la asignatura no estaba todavía disponible.

Fredrickson, B. L. (2001). The role of positive emotions in positive psychology. The broaden-and-build theory of positive emotions. American Psychologist, 56, 218-226.

llardi, B. C., Leone, D., Kasser, T. y Ryan, R. M. (1993). Employee and supervisor ratings of motivation: main effects and discrepancies associated with job satisfaction and adjustment in a factory setting. Journal of Applied Social Psychology, 23, 1.789-1.805.

Kahu, E. R. (2013). Framing student engagement in higher education. Studies in Higher Education, 38, 758-773.

Kasser, V. y Ryan, R. M. (1999). The relation of psychological needs for autonomy and relatedness to vitality, well-being, and mortality in a nursing home. Journal of Applied Social Psychology, 29, 935-454.

Kowal, J. y Fortier, M. S. (1999). Motivational determinants of flow: contributions from self-determination theory. The Journal of Social Psychology, 139, 355-368.

Marks, H. M. (2000). Student engagement in instructional activity: patterns in the elementary, middle, and high school years. American Educational Research Journal, 37, 153-184.

Ntoumanis, N. (2001). A self-determination approach to the understanding of motivation in physical education. British Journal of Educational Psychology, 71, 225-242.

Podsakoff, P. M., MacKenzie, S. B., Lee, J. Y. y Podsakoff, N. P. (2003). Common method biases in behavioural research: a critical review of the literature and recommended remedies. Journal of Applied Psychology, 88, 879-903. 
Reeve, J. (2012). A self-determination theory perspective on student engagement. En S. L. Christenson, A. L. Reschly y C. Wylie (Eds.), Handbook of Research on Student Engagement (pp. 149-172). New York: Springer.

Reis, H. T., Sheldon, K. M., Gable, S. L., Roscoe, J. y Ryan, R. M. (2000). Daily well-being: the role of autonomy, competence, and relatedness. Personality and Social Psychology Bulletin, 26, 419-435.

Salanova, M., Schaufeli, W. B., Xanthopoulou, D. y Bakker, A. B. (2010). The gain spiral of resources and work engagement: sustaining a positive worklife. Work Engagement: A Handbook of Essential Theory and Research, 118-131.

Seifert, T. (2004). Understanding student motivation. Educational research, 46, 137-149.

Seligman, M. E. P. y Csikszentmihalyi, M. (2000). Positive psychology: an introduction. American Psychologist, 55, 5-14.

Sheldon, K. M., Ryan, R. M. y Reis, H. T. (1996). What makes for a good day? Competence and autonomy in the day and in the person. Personality and Social Psychology Bulletin, 22, 1.270-1.279.
Spector, P. E. y Brannick, M. T. (2010). Common method issues: an introduction to the feature topic in organizational research methods. Organizational Research Methods, 13, 403-406.

Standage, M., Duda, J. L. y Ntoumanis, N. (2005). A test of self-determination theory in school physical education. British Journal of Educational Psychology, 75, 411-433.

Steers, R. M., Mowday, R. T. y Shapiro, D. L. (2004). Introduction to special topic forum: the future of work motivation theory. The Academy of Management Review, 29, 379-387.

Trowler, V. y Trowler, P. (2010). Student Engagement Evidence Summary, York, UK: Higher Education Academy.

Vallerand, R. J. y Bissonnette, R. (1992). Intrinsic, extrinsic, and amotivational styles as predictors of behavior: $A$ prospective study. Journal of Personality, 60, 599-620.

Vroom, V. H. (1964). Work and Motivation. New York: Wiley.

Walker, C. O., Greene, B. A. y Mansell, R. A. (2006). Identification with academics, intrinsic/extrinsic motivation, and self-efficacy as predictors of cognitive engagement. Learning and Individual Differences, 16, 1-12.

\section{APÉNDICE}

\section{Escalas de medida}
A) Escala de satisfacción de las necesidades psicológicas básicas
Autonomía
En esta clase....:
1. Puedo decidir las actividades a realizar.
2. Puedo opinar sobre las habilidades que quiero adquirir.
3. Siento que estoy estudiando Dirección de Empresas porque realmente quiero.
4. Me siento forzado a realizar las actividades que propone el profesor $(R)$. 


\section{A) Escala de satisfacción de las necesidades psicológicas básicas}

\section{Autonomía}

.......

5. Siento que tengo cierto grado de libertad de acción.

6. Siento que puedo escoger sobre lo que quiero hacer en esta clase.

\section{Competencia}

7. Creo que soy bastante bueno en Dirección de Empresas.

8. Hasta el momento, estoy satisfecho con mis resultados en Dirección de Empresas.

9. Cuando llevo un rato en la clase de Dirección de Empresas, me siento bastante competente en esta área.

10. Estoy muy formado en Dirección de Empresas.

11. No soy capaz de entender muy bien Dirección de Empresas (R).

\section{Relaciones}

Con mis compañeros de la clase de Dirección de Empresas me siento...:

12. Apoyado.

13. Comprendido.

14. Escuchado.

15. Valorado.

16. Seguro.

\section{B) Escala de motivación intrínseca}

Me involucro en las actividades que propone el profesor...:

17. Porque Dirección de Empresas es divertida.

18. Porque disfruto aprendiendo cosas nuevas.

19. Porque Dirección de Empresas es interesante.

20. Por la sensación que obtengo al aprender destrezas/técnicas nuevas.

\section{C) Escala de motivación extrínseca}

Me involucro en las actividades que propone el profesor...:

21. Porque, si no lo hago, se me penalizará en la nota final. 


\section{C) Escala de motivación extrínseca}

$$
\ldots / \ldots
$$

22. Porque es lo que se supone que tengo que hacer.

23. Para que el profesor no me llame la atención.

24. Porque son las reglas de esta clase.

\section{D) Escala de bienestar}

En esta clase de Dirección de Empresas me siento...:

25. Feliz.

26. Satisfecho.

27. Emocionado.

28. Relajado.

29. Orgulloso.

\section{E) Escala de implicación académica}

En esta clase de Dirección de Empresas...:

30. ¿Con qué frecuencia te esfuerzas tanto como puedes?

31. ¿Con qué frecuencia realizas las actividades propuestas por el profesor?

32. ¿Con qué frecuencia prestas atención en esta clase?

33. ¿Con qué frecuencia te aburres en esta clase? (R)

Nota. $R=$ reverse code. 